EPJ Web of Conferences 41, 09007 (2013)

DOI: $10.1051 /$ epjconf/20134109007

(C) Owned by the authors, published by EDP Sciences, 2013

\title{
Third- and fifth-harmonic generation by mid-infrared ultrashort pulses: beyond the fifth-order nonlinearity
}

\author{
D. Kartashov ${ }^{1}$, S. Ališauskas ${ }^{1}$, A. Pugžlys ${ }^{1}$, A.A. Voronin ${ }^{2}$, A.M. Zheltikov ${ }^{2,3}$, and A. Baltuška ${ }^{1}$ \\ ${ }^{1}$ Photonics Institute, Vienna University of Technology, Gusshausstrasse 27-387, A-1040 Vienna, \\ Austria \\ ${ }^{2}$ Physics Department, International Laser Center, M.V. Lomonosov Moscow State University, \\ 119992 Moscow, Russia \\ ${ }^{3}$ Department of Physics and Astronomy, Texas A\&M University, College Station TX, 77843-4242, \\ USA
}

\begin{abstract}
Third- and fifth-harmonic generation by ultrashort laser pulses in the midinfrared reveals nonlinear-optical effects beyond the fifth-order nonlinearity and enables, due to an extraordinarily long coherence length, efficient multiplex frequency upconversion of ultrashort mid-IR pulses.
\end{abstract}

Harmonic generation is one of the fundamental effects in nonlinear optics [1]. This phenomenon is at the heart of efficient frequency conversion schemes [2], high-resolution microscopy [3], and advanced attosecond technologies [4]. While high-order harmonic generation in the strong-field regime enables the synthesis of unprecedentedly short field waveforms, low-order harmonics are used for nonperturbing measurements on atomic and molecular systems, giving an access to the key parameters of materials, including their optical susceptibilities. The emerging field of ultrafast nonlinear optics in the mid-infrared $[5,6]$ calls for a deeper understanding of optical nonlinearities in the mid-IR. The first experiments on the filamentation of ultrashort mid-IR pulses [7] reveal new effects and unusual regimes, suggesting new physics behind the ultrafast nonlinearoptical dynamics of field waveforms in this spectral range and requiring generalization of the existing models of shortpulse evolution. The latest breakthroughs in the development of mid-IR sources capable of delivering sub-100-fs pulses with wavelengths well beyond $3 \mathrm{~mm} \mathrm{[5]} \mathrm{open} \mathrm{unique} \mathrm{possibilities} \mathrm{for}$ in-depth studies of optical nonlinearities in the mid-IR, allowing low-order optical harmonics to be generated in the regime of weak dispersion and, hence, extraordinarily large interaction lengths.

Here, we experimentally demonstrate and theoretically analyze third- and fifth-harmonic generation by 80 -fs pulses of $3.9-\mu \mathrm{m}$ radiation with a peak power up to $0.15 \mathrm{TW}$ in the regime of collimated beams. Experiments were performed with a hybrid OPCPA high-power femtosecond laser system delivering $20-\mathrm{Hz}, 80-\mathrm{fs}$ laser pulses at $3.9 \mu \mathrm{m}$ with an energy up to $12 \mathrm{~mJ}$ [5]. A collimated-beam output of this system with a beam diameter of $12 \mathrm{~mm}$ was used to generate the third and fifth optical harmonics along a straight propagation path in a gas medium. High-resolution infrared and visible-UV spectrometers were used to measure the spectra of the third and fifth harmonics, covering the spectral ranges of $1.2-1.7 \mu \mathrm{m}$ and $200-1100 \mathrm{~nm}$, respectively. The

This is an Open Access article distributed under the terms of the Creative Commons Attribution License 2.0, which permits unrestricted use, distribution, and reproduction in any medium, provided the original work is properly cited. 

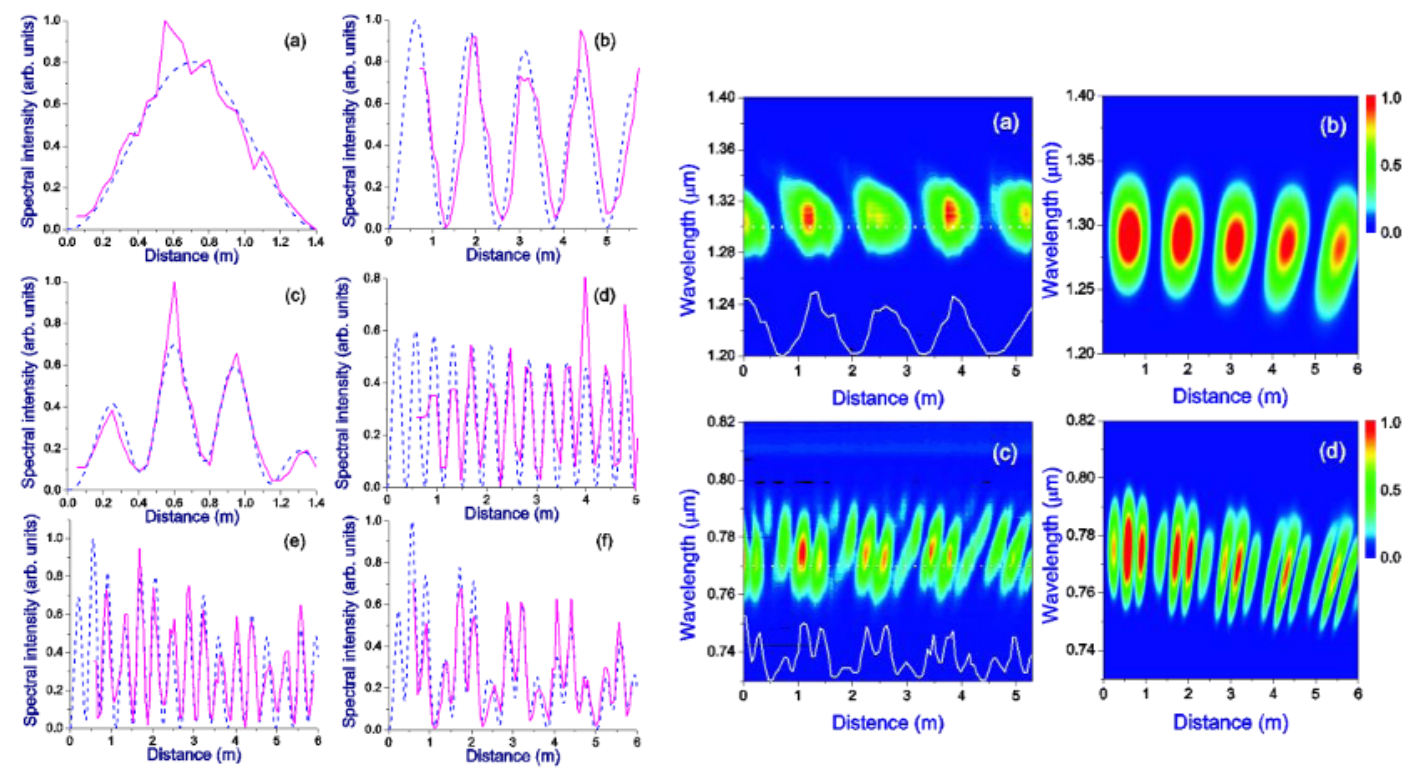

Fig. 1. (Left panel) Intensities of the third $(a, b)$ and fifth (c -- f) harmonics measured (solid lines) and calculated (dashed lines) as a function of the propagation path of 80 -fs mid-IR pump pulses in argon $(\mathrm{a}, \mathrm{c})$ and in the atmospheric air (b, d -- f). The pump energy is $4 \mathrm{~mJ}(\mathrm{~d}), 6.5 \mathrm{~mJ}(\mathrm{e}), 9 \mathrm{~mJ}(\mathrm{a}-\mathrm{c}$, f). (Right panel) The spectra of the third $(a, b)$ and fifth $(c, d)$ harmonics measured $(a, c)$ and calculated $(b, d)$ for different propagation paths of the pump field in the atmospheric air. The harmonic spectra at a fixed propagation path (vertical lines in the map) are shown on the right of the experimental maps.

nonlinear interaction length was varied with a 3-mm-thick BK7 plate inserted in the optical path, providing $90 \%$ attenuation of the mid-IR light. The intensity of optical harmonics was measured as a function of the interaction length by scanning this plate along the beam.

Results of third- and fifth-harmonic generation experiments in argon and atmospheric air are presented in Fig. 1. Intensities of the third and fifth harmonics of $80-\mathrm{fs}, 3.9-\mu \mathrm{m}$ pump pulses measured as a function of the propagation length display clearly resolved, high-visibility fringes, indicating large coherence lengths for harmonic generation processes. The slope of harmonic traces in the right panel of Fig. 1 reflects the wavelength dependence of the coherence length of harmonic generation processes, associated with gas dispersion.

Our model of harmonic generation by ultrashort mid-IR pulses is based on the numerical solution of the generalized Schrödinger equation for the electric field, which has been adapted for the purposes of this work to include the terms describing third- and fifth-harmonic generation:

$$
\frac{\partial \tilde{E}}{\partial z}=[i \widehat{D}(\omega)-\alpha(\omega)] \tilde{E}+\frac{i \omega}{2 c} \hat{F}\left\{\sum_{m=1}^{4} \chi^{(2 m+1)} E^{(2 m+1)}\right\}
$$

Here $z$ is the coordinate along the propagation direction, $\tilde{E}=\tilde{E}(\omega, z)$ is the Fourier transform of the electric field $E=E(\eta, z), \eta$ is the time in the retarded frame of reference, $\omega=2 \pi c / \lambda$ is the frequency, $\hat{F}$ is the Fourier transform operator, $\widehat{D}=k(\omega)-k\left(\omega_{0}\right)-\partial k /\left.\partial \omega\right|_{\omega=\omega_{0}}\left(\omega-\omega_{0}\right)$ is the dispersion operator, $\omega_{0}$ is the central frequency of the input field, $k(\omega)=\omega n(\omega) / c, n(\omega)$ is the refractive index, $\alpha(\omega)$ is the absorption coefficient, $c$ is the speed of light in vacuum, and $\chi^{(m)}$ is the $m$ th-order nonlinear susceptibility. Along with direct and cascade harmonic generation processes, Eq. (1), written for the electric field, includes self- and cross-phase modulation effects, as well as pulse self-steepening. Gas dispersion and absorption has been included through the Sellmeier 
formula for argon and through the Mathar model [8] with the use of the HITRAN database [9] in the case of atmospheric air.

The most striking feature of the fifth-harmonic signal seen in the left panel of Figs. 1 is that the waveform of its fringes depends on the pump intensity. This behavior is inconsistent with the canonical solution to the slowly varying envelope equation for the fifth harmonic [2] including only $\chi^{(3)}$ and $\chi^{(5)}$ terms, which predicts the $I^{5}$ scaling for both direct and cascaded fifth-harmonic terms with the pump intensity $I$. This intensity dependence of the waveform of the fifth-harmonic fringes cannot be explained in terms of a perturbative treatment of the nonlinear polarization including only the $\chi^{(3)}$ and $\chi^{(5)}$ terms, directly indicating the influence of high-order optical nonlinearities and suggesting a method for measuring high-order nonlinear-optical susceptibilities. The minimum set of nonlinear-optical susceptibilities found from the best fit of the numerical solution of Eq. (1) for the experimental results is $\chi^{(3)}=1.1 \cdot 10^{-21} \mathrm{~cm}^{2} / \mathrm{V}^{2}, \chi^{(5)}=3.1 \cdot 10^{-36} \mathrm{~cm}^{4} / \mathrm{V}^{4}, \chi^{(7)}=-1.0 \cdot 10^{-50} \mathrm{~cm}^{6} / \mathrm{V}^{6}$, and $\chi^{(9)}=-1.8 \cdot 10^{-67} \mathrm{~cm}^{8} / \mathrm{V}^{8}$.

In summary, third- and fifth-harmonic generation by ultrashort mid-IR pulses, enhanced due to an extraordinarily long coherence length, reveals nonlinear-optical effects beyond the fifth-order nonlinearity. With the wavelengths of the sevenths and ninth optical harmonics of the 4- $\mu \mathrm{m}$ pump also falling within the transparency range of a broad variety of atomic and molecular gases and allowing comfortable detection, the collimated-beam geometry of harmonic generation demonstrated in this work can be readily generalized to the metrology of nonlinear susceptibilities of even higher orders.

\section{References}

1. N. Bloembergen, Nonlinear optics (Benjamin, Reading, MA, 1977).

2. J.F. Reintjes, Nonlinear Optical Parametric Processes in Liquids and Gases (Orlando: Academic, 1984).

3. J.A. Squier, M. Müller, G.J. Brakenhoff, and K.R. Wilson, Opt. Express 3, 315 - 324 (1998).

4. P. B. Corkum and F. Krausz, Nature Phys. 3, 381 - 387 (2007).

5. G. Andriukaitis, T. Balciunas, S. Alisauskas, A. Pugžlys, A. Baltuška, T. Popmintchev, M.-C. Chen, M. M. Murnane, and H. C. Kapteyn, Opt. Lett. 36, 2755 - 2757 (2011).

6. G.O. Ariunbold, P. Polynkin, and J.V. Moloney, Opt. Express 20, 1662-1667 (2012)

7. D. V. Kartashov, S. Alisauskas, A. Pugžlys, A. Baltuška, A. Voronin, A.M. Zheltikov, M. Petrarca, P. Bejot, J. Kasparian, Ultrafast Optics (UFO VIII), Monterey, California (2011), pp. $71-72$.

8. R.J. Mathar, Appl. Opt. 43, 928 - 932 (2004)

9. http://www.cfa.harvard.edu/hitran/ 